\title{
14 Der Streit um die Konversation zwischen Charles-Augustin Sainte-Beuve und Marcel Proust
}

\subsection{Literaturkritik als ,causerie': die ,l'homme-et-l'œuvre'-Forschung bei Sainte-Beuve}

Charles-Augustin Sainte-Beuve, der bekannteste Literaturkritiker des 19. Jahrhunderts, gilt in der Literaturgeschichtsschreibung als einer der Urheber der biographischen literaturwissenschaftlichen Methode. Details aus dem Leben und Umfeld des Autors werden herangezogen, um dessen literarische Texte besser verstehen zu können. In einzelnen Fällen entwickelt und verengt sich diese Methode zu einem Biographismus, der Figuren oder Szenen aus der Literatur direkt mit Ereignissen und Begegnungen aus der Biographie des Autors zu erklären sucht. In der akademischen Literaturwissenschaft Frankreichs war die biographische literaturwissenschaftliche Methode als ,l'homme et l'œuvre-Forschung' bis in die achtziger Jahre hinein eine besonders prominente Form der Annäherung an literarische Texte und ihren Autor ${ }^{1}$. Bis zur Abschaffung der sogenannten ,thèse d'état', der Qualifikationsschrift für die Berufung auf eine Universitätsdozentur bzw. Professur und der Einführung der ,habilitation‘ 1984 war sie die geläufige Form der Beschäftigung mit der Literatur, bei der ihre Verfasser über einen Zeitraum von oftmals bis zu zehn Jahren alles Auffindbare zur Biographie eines Schriftstellers zusammentrugen, um dieses Material dann in der ein oder anderen Form mit den Texten in Verbindung zu bringen. In seiner Streitschrift Contre Sainte Beuve, die 1909 verfasst, aber erst 1954 posthum veröffentlicht wird, kritisiert Marcel Proust die Methode Sainte-Beuves, das Werk durch die Brille der Biographie des Autors zu lesen, bzw. im Werk den Autor und seine Lebenswelt aufzuspüren (,[...] cette méthode qui consiste à ne pas séparer l'homme et l'œuvre [...]“2) als positivis-

1 Vgl. dazu grundlegend José-Luis Diaz, L'homme et l'œuvre. Contribution à une histoire de la critique (Les Littéraires), Paris, PUF, 2011.

2 Vgl. die Ausgabe Marcel Proust, Contre Sainte-Beuve suivi de Nouveaux Mélanges, hrsg. von Bernard de Fallois (nrf), Paris, Gallimard 1954, S. 136. Die Seitenangaben im laufenden Text beziehen sich auf diese Ausgabe. Zur Problematik der Textedition dieses Roman-Essais vgl. das Nachwort in der deutschen Ausgabe von Mariolina Bongiovanni Bertini und Luzius Keller: Marcel Proust, Gegen Sainte-Beuve (= Werke. Frankfurter Ausgabe. Bd. 3), Frankfurt a. M., Suhrkamp, 1997, S. 315-336. Vgl. auch die Ausgabe: Contre Sainte-Beuve, in: M. P., Contre Sainte-Beuve, précédé de Pastiches et Mélanges et suivi de Essais et articles, hrsg. von Pierre Clarac und Yves Sandre (Bibliothèque de la Pléiade. 229), Gallimard, 1971, S. 209-312, hier: S. 221.

Ә Open Access. (c) 2021 Michael Bernsen, publiziert von De Gruyter (c) BY-NC-ND Dieses Werk ist lizenziert unter der Creative Commons Attribution 4.0 Lizenz.

https://doi.org/10.1515/9783110758009-014 
tisch und oberflächlich. Für ihn ist das literarische Werk das Ergebnis eines völlig anderen als des gesellschaftlichen Autor-Ichs: ,[...] un livre est le produit d'un autre moi que celui que nous manifestons dans nos habitudes, dans la société, dans nos vices.“ (S. 136) Man könne den Verfasser von Versen nicht mit dem Gesprächsteilnehmer einer Salonkonversation verrechnen.

Vor allem der Hinweis auf die Salonkonversation gibt zu erkennen, aus welchem Kontext heraus die literaturkritische Methode Sainte-Beuves laut Proust entstanden ist. Der Kritiker verfasst zwischen 1849 und 1869 biographisch-essayistische Abhandlungen zur Literatur, die in diversen Pariser Zeitungen jeweils Montags veröffentlicht und 1851-1862 bzw. 1863-1870 unter den Titeln Causeries du lundi und Nouveaux lundis auch in Buchform gedruckt vorliegen. Seine Portraits littéraires (1844) und die Portraits contemporains (1846) komplettieren diese aus dem geselligen Kontext der Konversation erwachsenen bzw. diesen simulierenden literaturkritischen Essays. Nicht allein, dass der Begriff der ,causeries' auf die Salons und den Geselligkeitskontext der Konversation verweist; die ,causerie ‘ liefert das Modell der literaturkritischen Methode, welches Louis Véron, der Direktor des Constitutionnel, anführt, als er 1849 das neue Format des montäglichen Feuilletons vorstellt, in dem Sainte-Beuve seine Literaturkritiken veröffentlicht:

Le temps des systèmes est passé, même en littérature. Il s'agit d'avoir du bon sens, mais de l'avoir sans fadeurs, sans ennui, de se mêler à toutes les idées pour les juger ou du moins pour en causer avec liberté et décence. C'est cette causerie que nous voudrions favoriser, et que M. Sainte-Beuve essaiera d'établir entre ses lecteurs et lui. ${ }^{3}$

Dass das Feuilleton des Constutionnel eine Diskussion zwischen dem Kritiker und seinen Lesern über Literatur lancieren will, ist auf den ersten Blick nachvollziehbar. In Zeiten einer größeren Öffentlichkeit verlieren die traditionellen Salons an Bedeutung, da sie nur kleinen Gruppen der Gesellschaft vorbehalten sind. Das Feuilleton soll die Salons ersetzen und die traditionelle ,causerie' auf einem anderen Niveau und unter Beteiligung eines neuen Mediums in eine größere Öffentlichkeit überführen. Inwiefern jedoch die literaturkritische Methode Sainte-Beuves der ,causerie‘ verpflichtet ist, ist erklärungsbedürftig.

An einem prominenten Beispiel lässt sich zeigen, wie Sainte-Beuve seine Literaturkritik aus der Salonkonversation ableitet und seine literaturkritische Methode sowie seinen Stil an der ,causerie‘ ausrichtet. Dabei zeigt sich auch, dass die

3 Abgedruckt in: Causeries du Lundi, 15 vol., Paris, Garnier, ${ }^{3} 1857-1870\left({ }^{1} 1851-1862\right)$, Bd. 1, S. 4. Vgl. auch Roxana Verona, Les „Salons“ de Sainte-Beuve. Le critique et ses muses (Romantisme et modernité. 28), Paris, Champion, 1999, S. 26 sowie Anne Martin-Fugier, „Le salon XVIIe siècle selon Sainte-Beuve“, Les Cahiers du Centre de Recherches Historiques H. 28-29/2002, S. 1-11, hier: S. 2. (http://journals.openedition.org/ccrh/1012). 
spezielle ,l'homme et l'œuvre'-Philologie Sainte-Beuves ganz von der Kunst des Impliziten durchdrungen ist und somit auch dieses so zentrale Element der französischen Konversation aufgreift. Am 5. und am 12. Mai 1862 erscheint unter dem Titel Madame de Staël. Coppet et Weimar, par l'auteur des Souvenirs de $M^{\text {me }}$ Récamier eine Rezension in zwei Teilen einer kommentierten Sammlung von Briefen Mme de Staëls an Luise von Weimar und an Madame Récamier. Die Besprechung dieser von Amélie Lenormant edierten Ausgabe findet zwei Jahre später Eingang in die Sammlung der Nouveaux lundis. Weit mehr als nur eine Rezension der Ausgabe lässt Sainte-Beuve hier geradezu mustergültig erkennen, worauf es ihm als Literaturkritiker ankommt und nach welchen Prinzipien er arbeitet. Gleich zu Beginn der eher als Porträt der Mme de Staël denn als Besprechung angelegten Auseinandersetzung stellt er eine Eingangsfrage, auf die er am Ende zurückkommt: Aufgrund welcher Erinnerungen überlebt ein Schriftsteller in der Gedankenwelt der Nachfahren? Für Sainte-Beuve ist klar, dass nicht allein die Schriften die Erinnerung bestimmen, sondern vor allem der Gesamteindruck, den man sich von einem Autor macht. Und bei der Erzeugung und Übermittlung dieses Gesamteindrucks spielt der Kritiker eine herausragende Rolle. Die Schriften der Mme de Staël drohen zu verblassen, so heißt es am Ende der Besprechung, zumal ihre Sätze und ihre vagen Ausdrücke vor den Augen der Akademie ohnehin kaum Bestand hätten.

Les souvenirs qui l'avaient accompagnée jusqu'ici cessent et expirent; les écrits seuls sont là désormais, et ils ont besoin d'être complétés, d'être expliqués: le plus fort de leur charme et de leur puissance est dans l'ensemble, et on ne saurait presque en détacher une page entre toutes. Les phrases même tiennent peu, prises en détail ; [...] A l'Académie [...] on allègue tantôt le vague de l'expression, tantôt l'impropriété des termes $[\ldots]^{4}$

Um Mme de Staël richtig zu würdigen, bedürfen ihre Schriften einer ganz besonderen Auslegung, die der Kritiker ganz woanders sucht und findet:

[...] le plus sûr est de ne pas s'en tenir uniquement aux écrits déjà anciens et qui ont jeté leur feu; le meilleur coup de fortune pour une mémoire immortelle est d'avoir, du sein du tombeau, deux ou trois de ces retours et de ces réveils magnifiques qui étonnent les générations nouvelles, qui les convainquent qu'un mort puissant est là, redoutable encore jusque dans son ombre et son silence ${ }^{5}$

4 „Madame de Staël. Coppet et Weimar par l'auteur des souvenirs de $\mathrm{M}^{\mathrm{me}}$ Récamier (suite et fin)“, in: Nouveaux lundis, 13 Bde., Paris, Lévy, 1864-1870, S. 312-332, hier: S. 331.

5 „Madame de Staël. Coppet et Weimar par l'auteur des souvenirs de $\mathrm{M}^{\mathrm{me}}$ Récamier (1)“, in: Nouveaux lundis, Bd. 2, S. 290-311, hier: S. 291. 
Sainte-Beuves Auseinandersetzung mit Mme de Staël versteht sich als ein solcher „réveil magnifique“, als einer jener „retour“, der die folgenden Generationen ehrfürchtig vor der Leistung der Autorin erstarren lässt. Bewundernswert werden die literarischen Äußerungen Mme de Staëls erst durch den Blick des Kritikers, der das Umfeld ihrer Schriften ausleuchtet und sie somit um eine nicht immer klar zu definierende Dimension bereichert.

[...] c'est à la condition d'y mettre [...] l'éclair du regard, la physionomie, l'accent; il faut tout cela à sa parole pour se complèter; sa plume n'avait pas ce qui termine: il manque presque toujours à sa phrase écrite je ne sais quel accompagnement.

C'est peut-être un motif de plus, pour le lecteur distingué, de s'y plaire, en y remettant partout cet air et cet accent sous-entendus. Les délicats aiment à avoir de ces occasions de placer leur délicatesse. ${ }^{6}$

Die Entschlüsselung des Impliziten, das Erspüren des Tons, der physiognomische Blick sind nun Verfahren, die Sainte-Beuve der Konversation der Salons entnimmt. Der Kritiker war selbst ständiger Gast mehrerer Salons der Zeit, im zweiten Kaiserreich insbesondere im Salon der Princesse Mathilde Bonaparte. Er legitimiert seine Ausführungen zu Autoren unmittelbar vorangegangener Epochen nicht nur mit der Lektüre von Memoiren, sondern auch mit Erfahrungen aus der ,causerie‘ mit Zeitzeugen, die er in diversen Gesprächen gewonnen habe:

J'ai souvent pensé, qu'un homme de notre âge qui a vu le premier Empire, la Restauration, le règne de Louis-Philippe, qui a beaucoup causé avec les plus vieux des contemporains de ces diverses époques, qui, de plus, a beaucoup lu de livres d'histoire et de Mémoires qui traitent des derniers siècles de la monarchie, peut avoir en soi, aux heures où il rêve et où il se reporte vers le passé, des souvenirs presque continus qui remontent à cent cinquante ans et au-delà. ${ }^{7}$

Besonders wichtig sind für Sainte-Beuve jedoch die Erfahrungen, die sich auf die Art der Konversation beziehen. Fasziniert ist er vor allem von jenen Übergängen zwischen der mündlichen Kommunikation und der literarischen Arbeit, insbesondere schreibender Frauen, da diese ja oftmals die Gastgeberinnen der Salons sind:

C'est chez elles [= les femmes], parmi celles qui ont écrit, qu'on trouverait le plus sûrement des témoignages de cette familiarité décente, de cette moquerie fine, et de cette aisance à tout dire, qui remplit d'autant plus les conditions des anciens qu'elles-mêmes n'y songeaient pas. $^{8}$

6 S. 332.

7 „Journal du Marquis de Dangeau“, in: Causeries du lundi, Bd. 11, S. 6.

8 „MM $\mathrm{M}^{\mathrm{me}}$ de Caylus et de ce qu'on appelle Urbanite““, in: Causeries du lundi Bd. 3, S. 56-77, hier: S. 70. 
Von Mme de La Fayette heißt es, bei ihr sei der Übergang der Konversation in die Literatursprache und ihre Schreibweise mit Händen zu greifen:

Cette personne n'écrivit [...] qu'assez peu, à son loisir, par amusement et avec une sorte de négligence qui n'avait rien du métier [... $]^{9}$

Im Porträt der Mme du Deffand interessiert er sich für ihren Schreibstil, der den Ton, die Lebendigkeit, die Energie und die Einfachheit der Konversation spiege$\mathrm{le}^{10}$. Für Anne Martin-Fugier, die sich mit Sainte-Beuves Einstellung zum Salon des 17. und 18. Jahrhunderts befasst, ist der Kritiker selbst zum Maßstab der Übertragung der ,causerie‘ auf die Literatur geworden. Die 1857 verfasste Histoire de la conversation von Émile Deschanel beruft sich fortwährend auf Sainte-Beuves Einschätzungen, so dass der viel bemühte Topos vom Untergang der Konversation im geschäftigen 19. Jahrhundert ein schiefes Licht auf die wahren Verhältnisse wirft:

Il serait plus juste de dire que le XIXe siècle ne cesse de s'interroger sur la sociabilité et la conversation, sur le changement des conditions du développement de l'urbanité, et, plus largement, sur ce qui fait l'identité française. ${ }^{11}$

In diesem medialen Übergangsprozess spielt Saint-Beuve für Martin-Fugier eine entscheidende Rolle:

Le XIXe siècle, passant d'un modèle aristocratique à une société en voie de démocratisation, a comme embaumé l'intimité idéale, faite de conversation, de correspondance et de sociabilité, telle qu'on imaginait qu'elle se pratiquait dans les siècles précédents, comme pour garder trace d'un paradis perdu. Sainte-Beuve a joué un rôle essentiel dans ce processus. ${ }^{12}$

In einer Salonkonversation liegt ein besonderes Interesse darin, seine Gesprächspartner einschätzen zu können. Anhand von Vorinformationen mündlicher oder schriftlicher Art bringt auch die unsystematischste ,causerie' zwangsläufig die Analyse der Äußerungen des Gegenübers mit sich. Beziehungen zu weiteren Personen spielen für die Einschätzung des Gesprächspartners eine wichtige Rolle. Darüber hinaus ist die Stimme sowie die gesamte Körpersprache von ent-

\footnotetext{
9 „Madame La Fayette“, in: Portraits de femmes (= Euvres. Bd. 2), hrsg. von Maxime Leroy (Bibliothèque le la Pléiade. 88), Paris, Gallimard 1951, S. 1206-1241, hier: S. 1206.

10 Dies geht zurück auf ein Zitat von Mme du Deffand: ,[...] je veux le ton de la conversation, de la vivacité, de la chaleur, et, par-dessus tout, de la facilité, de la simplicité.“ („Lettre de la Marquise du Deffand“, in: Causeries du lundi, Bd. 1, S. 412-431, hier: S. 428. Vgl. auch Martin-Fugier, „Le Salon VIIe siècle“, S. 4)

11 Martin-Fugier, „Le Salon VIIe siècle“, S. 8.

12 Vgl. ebd.
} 
scheidender Bedeutung für den Gesamteindruck von der Person. Im Besonderen geht es darum, jene Verhaltensprinzipien zu ergründen, in denen wichtige beobachtete Verhaltensweisen sowie Einstellungen der Person zusammenlaufen. Sainte-Beuve ist allein aufgrund seiner Geschichte des Klosters von Port-Royal (Port-Royal, 1840-1848) mit den Anschauungen der Moralistik vertraut und in der moralistischen Beobachtungsgabe geschult. Da sich der Mensch nach moralistischer Auffassung aufgrund seiner umtriebigen Eigenliebe schwer auf eine Position festlegen lässt, erfordert die Analyse des Gesprächspartners eine hohe Sensibilität und ein intuitives Urteilsvermögen. Die moralistische Analyse eines Gesprächspartners bedingt auf doppelte Weise die Kunst des Impliziten: Der Mensch - so sieht es die negative Anthropologie der Moralistik - ist rätselhaft, so dass sich die hinter seinem Verhalten liegenden Motivationen nur auf implizite Weise äußern. Auf der anderen Seite ist jedoch auch die Annäherung an diese Verhaltensweisen nur qua ,via obliqua', auf implizite Weise, möglich. Im Spiel der ,causerie', dem spielerischen Herantasten an den Gesprächspartner ergeben sich plötzliche Einsichten, Evidenzen verborgener Bewusstseinsschichten. Die Gesprächspartner müssen somit die Kunst des Impliziten beherrschen: Sie nähern sich sozusagen auf einem doppelbödigen, indirekten Weg aneinander an, um einen Eindruck der maßgeblichen Antriebskräfte des Gegenübers zu gewinnen.

Diese Überlegungen liegen der literaturkritischen Methode Sainte-Beuves zugrunde. Wie in einem Gespräch tastet er sich ausgehend von den Texten der Autoren, denen er im Einzelfall gar nicht die zentrale Stellung beimisst, an die Person des Autors und sein Umfeld heran. Im 19. Jahrhundert werden nicht mehr die Briefe eines Autors oder einer Autorin in Salons gelesen und diskutiert, sondern Briefsammlungen herausgegeben und in der Presse vorgestellt ${ }^{13}$. Saint-Beuve bespricht solche Briefsammlungen, indem er wie im oben angeführten Fall der Mme de Staël weitere Zeugnisse der Zeit zu Zwecken der Dokumentation heranzieht. Der Kritiker bringt somit unterschiedliche Stimmen aus Briefen, Memoiren und anderen historischen Zeugnissen quasi in ein Gespräch miteinander und mit dem Autor:

Was Literatur ist, erfährt bei Sainte-Beuve keine normative Eingrenzung. Prinzipiell ist alles, was aus der Unmittelbarkeit der Kommunikation herausführt und die Festigkeit des schriftlichen Zeugnisses gewinnt, der Literatur zugehörig. Korrespondenz, Memoiren, Journale, Geschichten, Reflexionen, Traktate, Pamphlete, es gibt keine Form, worin der Geist nicht eine Sprache finden könnte, und überall, wo diese Sprache eigentümlich und klar vernehmbar

13 Vgl. dazu Martin Fugier, „Le Salon VIIe siècle“, S. 8 f. 
ist und in irgendeiner Weise den Spielraum der Möglichkeiten erweitert, der Vielgestaltigkeit des Lebens eine Sprache zu finden, wird Sainte-Beuves Interesse geweckt. ${ }^{14}$

Seine Vorgehensweise beschreibt der Kritiker selbst als „physiologie morale“15. In den programmatischen Bemerkungen seines ,portrait littéraire‘ von Chateaubriand heißt es:

La littérature, la production littéraire, n'est point pour moi distincte ou du moins séparable du reste de l'homme et de l'organisation; je puis goûter une œuvre, mais il m'est difficile de la juger indépendamment de la connaissance de l'homme même; et je dirais volontiers: tel arbre, tel fruit. L'étude littéraire me mène ainsi tout naturellement à l'étude morale. ${ }^{16}$

Im Zentrum dieser Physiologie steht die Suche nach dem neuralgischen Punkt, in dem das Werk und die Person des Autors zusammenfallen. Diese Suche nach der „qualité de son esprit“17, wie es im Porträt von Rivarol heißt, erfolgt oftmals intuitiv; sie ergibt sich gleichsam als jener „réveil magnifique“, von dem die ,causerie“ über Mme de Staël zu berichten weiß. Programmtisch wird dies im Porträt Diderots entwickelt. Sainte-Beuve versteht sich als Komponist der „biographie comparée“ des Autors:

J'ai toujours aimé les correspondances, les conversations, les pensées, tous les détails du caractère, des mœurs, de la biographie, en un mot, des grands écrivains; surtout quand cette biographie comparée n'existe pas déjà rédigée par un autre, et qu'on a pour son propre compte à la construire, à la composer. ${ }^{18}$

Wie in einer Konversation mit einem Gesprächspartner kristallisiert sich für Sainte-Beuve der Gesamteindruck aus Autor und Werk heraus, den er hier als „la perle“19 bezeichnet. In einem fortwährenden Dialog mit den Quellen entsteht ein kreativer Prozess wie in der Malerei bzw. der Bildhauerei, an dessen Ende ein

14 Karlheinz Stierle, „L'homme et l'œuvre. Sainte-Beuves Literaturkritik“, in: Wilfried Barner (Hrsg.), Literaturkritik - Anspruch und Wirklichkeit. DFG-Symposium 1989 (Germanistische-Symposien-Berichtsbände. 12), Stuttgart, Metzler, 1990, S. 185-196, hier: S. 189.

15 Vgl. Stierle, „L'homme et l'œuvre“, S. 188.

16 „Chateaubriand jugé par un ami intime en 1803 (Suite et fin)“, in: Nouveaux lundis Bd. 3, S. 15-33, hier: S. 15. Vgl. auch Stierle, „L'homme et l'œuvre“, S. 188.

17 „Rivarol“, in: Causeries du Lundi, Bd. 5, Paris 1944, S. 62-84, hier: S. 63. Vgl. Stierle, „L’homme et l'œuvre“, S. 189.

18 „Diderot“, in: Portraits littéraires (= Euvres. Bd. 1), hrsg. von Maxime Leroy (Bibliothèque le la Pléiade. 80), Paris, Gallimard 1949, S. 867-888, hier: S. 867.

19 S. 868. 
individuelles, gleichsam funkensprühendes, sprechendes Porträt steht, welches Widersprüche nicht verdeckt, sondern mit einem „entlarvenden Lächeln“ die Brücke zwischen den Ungereimtheiten sichtbar macht:

[...] on l'étudie, on le retourne, on l'interroge à loisir; on le fait poser devant soi; c'est presque comme si l'on passait quinze jours à la campagne à faire le portrait où le buste de Byron, de Scott, de Goethe; seulement on est plus à l'aise avec son modèle, et le tête-à-tête, en même temps qu'il exige un peu plus d'attention, comporte beaucoup plus de familiarité. Chaque trait s'ajoute à son tour, et prend place de lui-même dans cette physionomie qu'on essaye de reproduire; c'est comme chaque étoile qui apparait successivement sous le regard et vient luire à son point dans la trâme d'une belle nuit. Au type vague, abstrait, général, qu'une première vue avait embrassé, se mêle et s'incorpore par degrés une réalité individuelle, précise, de plus en plus accentuée et vivement scintillante; on sent naître, on voit venir la ressemblance; et le jour, le moment où l'on a saisi le tic familier, le sourire révélateur, la gerçure indéfinissable, la ride intime et douloureuse qui se cache en vain sous les cheveux déjà clairsemés, - à ce moment l'analyse disparaît dans la création, le portrait parle et vit, on a trouvé l'homme. II y a plaisir en tout temps à ces sortes d'études secrètes $[\ldots]^{20}$

Im Fall der Mme de Staël tun sich für Sainte-Beuve besonders viele Widersprüche auf: Mehr als das Werk, das oftmals im Vagen verbleibt und in Vergessenheit zu geraten droht, beeindruckt ihn die Person der Autorin mit ihren Widersprüchen, die es allerdings besonders schwer machen, die ,qualité de son esprit‘ bzw. die ,perle‘ herauszumodellieren. Die rezensierte Briefausgabe Mme de Staël. Coppet et Weimar spiegelt für Sainte-Beuve nur ein konventionelles Bild der Autorin wider. Und so zieht er weitere Briefe von Sismondi, Stellungnahmen von Schiller, Byron u. a. heran²1, Quellen, die ergeben, dass Mme de Staël auf die Äußerung von Gefühlen und Ideen gleichermaßen Wert legt, dass sie mit einer fortwährenden inquisitorischen Unruhe den Dingen nachgeht, dass sie trotz ihrer Großzügigkeit („générosité“"22) oftmals scharfzüngig und sogar beleidigend mit Gesprächspartnern umgeht, allein um ihre geistige Überlegenheit $\mathrm{zu}$ beweisen ${ }^{23}$. In all diesen

20 S. 867.

21, „[...] je veux rassembler [...] quelques témoignages qui tous s'accordent, mais qui sont cependant un peu plus variés de ton que ceux que je trouve réunis dans le livre de Coppet et Weimar." (,Madame de Staël”, S. 302)

22 S. 329.

23 So in einem Brief von Sismondi : „[...] excepté dans tous ses jugements elle est trop souvent haineuse et méprisante [...][la puissance] de sa réputation, qui s'est toujours plus confirmée, lui a fait contracter plusieurs des défauts de Bonaparte. Elle est comme lui intolérante de toute opposition, insultante dans la dispute, et très-disposée à dire aux gens des choses piquantes, sans colère et seulement pour jouir de sa supériorité.” (S. 303) 
Widersprüchen kristallisiert sich für Sainte-Beuve die interessante ,qualité de son esprit' heraus: Mme de Staël ist, so der Gesamteindruck, eine Theoretikerin der ,causerie“ und darüber hinaus zugleich die Verkörperung der Prinzipien der Konversation in Reinkultur: „Mme de Staël [...] définit [...] la conversation, [...] elle qui en était le Génie même [...]“24. Dabei lobt er besonders Mme de Staëls Kunst des Impliziten, des Vaguen, ohne die Dinge allzu sehr zu vertiefen, die Kunst der Erzeugung unerwarteter Effekte durch die Herstellung subtiler Bezüge:

Car elle voulait du sentiment aussi et avant tout, mêlé aux idées, avec des éclairs de gaieté fugitive, quantité de rapports fins, subtils, déliés, des anecdotes d'une application spirituelle et imprévue de soudains essors et comme des flammes vers les plus hauts sommets; mieux que des aperçus, des considérations politiques et historiques, fortement exprimées, mais sans s'y appesantir; des images même, qui peut-être n'auraient point paru des images en plein soleil, mais qui en faisaient l'effet dans un salon; puis tout à coup (car c'était une femme toujours) un soupir romanesque jeté en passant, et quelque perspective lointaine vaguement ouverte sur la destinée, les peines du cœur, les mystères de la vie; un coin mélancolique à l'horizon. C'est dans cet ensemble qu'elle excellait; c'est cette trame diverse et mobile qu'elle agitait, qu'elle variait et recommençait sans cesse avec un art de magicienne $[\ldots]^{25}$

Sainte-Beuve macht sich die Auffassung Mme de Staëls zu eigen, dass die Konversation in ihrer speziellen Form der ,causerie“ ein nationales Charakteristikum Frankreichs ist, ganz anders in Deutschland, wo es nur um den Sinn, das Resultat und die Schlussfolgerung gehe und wo man die Kunst des Impliziten, das Spiel des - scheinbar - Belanglosen nicht kenne:

[...] elle n'était proprement dans son milieu et dans son centre, dans sa pleine souveraineté, qu'en conversant et en causant. Or, elle l'a dit, on ne cause véritablement qu'en France et en francais : „la conversation, comme talent, n'existe qu'en France“. [... [ En Allemagne, on est trop occupé du but et du résultat sérieux, et dans toute discussion, de la conclusion même; on ignore l'art d'agir, de parler sans but et pourtant avec intérêt. ${ }^{26}$

Gegen diese Bestimmung der Konversation als geistreiche Umspielung des eigenen Interesses und somit als nationaltypisches Charakteristikum in Frankreich, welches auch die Literatur dominiert, erhebt sich zu Beginn des 20. Jahrhunderts erheblicher Widerspruch.

24 S. 309.

25 S. 310; Hervorhebungen von Vf.

26 S. $308 \mathrm{f}$. 


\subsection{Prousts Zurückweisung der ,causerie du salon“ in seiner Schrift gegen Sainte-Beuve}

Die Übertragung der Konversation auf die Literatur und in der Folge auch auf die Literaturkritik stößt auf vehemente Kritik bei Marcel Proust. Proust versucht, die Literatur radikal von der Konversation zu trennen. Allein die Literatur kann für ihn eine Tiefe garantieren, zu der die ,oberflächliche' Konversation nicht in der Lage ist. Die Literatur, und damit meint Proust in erster Linie die Schrift, ermöglicht es für ihn, in tiefere Bewusstseinsschichten vorzudringen, die die Konversation nicht erreichen kann, da sie an die soziale Geselligkeit und damit an die Stimme gebunden ist. Prousts Hauptwerk À la recherche du temps perdu lässt sich als eine durchgängige Auseinandersetzung mit der Konversation deuten. Wie kaum ein anderes Romanwerk bilden seine Romane die ganze Bandbreite der zeitgenössischen Salonkonversationen ab. Diese „Konversationsexzesse“ stellen das auffälligste Merkmal seiner Romane dar, welches dem Leser bei einem Autor, der nach eigenen Aussagen poetologisch ganz andere Ziele verfolgt, äußerst „befremdlich“ erscheinen muss:

[...] die Konversationsveranstaltungen, von denen die Recherche berichtet, [...] die Matinées, Soirées und Dîners der Recherche dehnen sich bekanntlich oft über mehr als hundert Seiten aus, und [...] so gewährt Proust den seinen das Privileg, sich in oft endlos anmutenden Äußerungen mehr oder weniger frei verströmen zu dürfen. Daß solche Ausführlichkeit der Konversations- und Rededarstellung befremdlich wirken muß, verraten aufs deutlichste die Reaktionen der Proust-Kritik [... ${ }^{27}$

Die Forschung zu Proust hat über einen langen Zeitraum angenommen, das poetologische Programm des Autors, welches er in dem frühen, 1909 verfassten, allerdings erst 1954 veröffentlichen hybriden Essay-Roman Contre Sainte-Beuve formuliert, werde in der Recherche umgesetzt. Die Konversationen würden nur deshalb so ausführlich abgebildet, um die Ziele der symbolistischen Poetik des Autors umso mehr zur Geltung zu bringen. Neuere Arbeiten zeigen jedoch, dass Proust das so nicht gelingt und dass er das letzten Endes auch gar nicht umsetzen will. Die symbolistische Poetik, die im literarischen Werk jenseits der sozialen Lebenssphäre, welcher die Konversation angehört, das von sozialen Fragen unberührte ,moi profond' ausloten und dem schriftstellerischen Kreationsprozess nachgehen will, wird von den akribischen Gedächtnisprotokollen der sozialen Konversationen geradezu überrollt:

27 Ulrich Schulz-Buschhaus, „Gemeinplatz und Salonkonversation bei Marcel Proust“, in: U. Sch.-B, Das Aufsatzwerk; http://gams.uni-graz.at/o:usb-06C-349, S. 6. 
Sowohl die mimetische Hingabe an das Kritisierte als auch die Differenziertheit der kommentierenden Stellungnahmen zeigen nun an, daß die Welt der Konventionen in Prousts Recherche bei aller Kritik nie ganz aus der Geborgenheit einer letzten Endes unversehrten auktorialen Sympathie entlassen wird. [...] Zwar weiß der Leser am Ende seiner Lektüre, daß in der gesellschaftlichen Welt der Recherche nichts Authentisches und Substantielles Bestand haben kann. Zugleich weiß er aber auch, daß diese Welt ohne Substanz und Authentizität - nach dem Beispiel der kritischen Empathie ihres Erzählers betrachtet - Aufmerksamkeit, Interesse und Zuwendung verdient hat wie keine andere. ${ }^{28}$

Auf diese Weise attestiert Proust der für die französische Kultur typischen Lust an der Konversation, der Kunst des Impliziten, eine grundlegende Bedeutung, auch wenn er sie einer profunden, satirischen Kritik unterzieht.

In seinem teils romanesken, teils essayistischen Werk Contre Sainte-Beuve wehrt sich Proust gegen die „méthode de Sainte Beuve“, das Werk eines Autors über die Heranziehung seiner Biographie zu erklären:

La littérature, disait Sainte-Beuve, n'est pas pour moi distincte ou du moins, séparable du reste de l'homme et de l'organisation [...] (S. 136)

Proust erklärt demgegenüber, dass man den sozialen Menschen strikt vom Ich des Schriftstellers trennen muss. Das soziale Ich, auf das Sainte-Beuve rekurriert, hat für Proust mit dem schreibenden Ich keine Gemeinsamkeiten. Deshalb bleiben die Kritiken Sainte-Beuves nur an der Oberfläche, ohne in die Tiefe der Werke einzudringen:

L'œuvre de Sainte-Beuve n'est pas une œuvre profonde. La fameuse méthode, qui en fait, selon Taine, selon Paul Bourget et tant d'autres, le maître inégalable de la critique du XIX ${ }^{e}$, cette méthode, qui consiste à ne pas séparer l'homme et l'œuvre, à considérer qu'il n'est pas indifférent pour juger l'auteur d'un livre, [...] cette méthode méconnaît ce qu'une fréquentation un peu profonde avec nous-mêmes nous apprend: qu'un livre est le produit d'un autre

28 Schulz-Buschhaus, „Gemeinplatz“, S. 15. Vgl. auch ders., „Topiken der Konversation bei Flaubert und Proust“, in: U. Sch.-B., Das Aufsatzwerk; http://gams.uni-graz.at/o:usb-06A-285. Vgl. dazu auch die einschlägige Dissertation von Ulrike Sprenger, die aus einer anderen Perspektive zum gleichen Befund gelangt. Prousts subjektorientierte Poetik der Schrift, die die Stimme der mündlichen Konversation zu überwinden trachtet, mündet - so Sprenger - letztlich in eine Inszenierung mündlicher Rede, deren Polyphonie das ursprüngliche, in der Schrift Contre SainteBeuve formulierte Ziel hinter sich lässt (Stimme und Schrift. Inszenierte Mündlichkeit in Prousts $A$ la recherche du temps perdu 5368, Tübingen, Narr, 1995). Zu ähnlichen Ergebnissen gelangt auch Pierre-Marie Héron, „Littérature et conversation au XXe siécle: Proust (encore)“, Revue d'histoire littéraire de la France Bd. 110/2010, S. 93-111. Vgl. auch das Kap. „Le Langage parlé“ bei Josee van den Ghinste, Rapports humains et communication dans À la recherche du temps perdu, Paris, Nizet, 1975, S. 139-195. 
moi que celui que nous manifestons dans nos habitudes, dans la société, dans nos vices. Ce moi-là, si nous voulons essayer de le comprendre, c'est au fond de nous-mêmes, en essayant de le recréer en nous, que nous pouvons y parvenir. (S. 136 f.)

Die Oberflächlichkeit der Beurteilung der Werke anhand der sozialen Umstände des Lebens eines Autors erklärt für Proust auch die krassen Fehlurteile SainteBeuves, der zahlreiche Autoren aus der zweiten Reihe ausführlich würdigt, den größten Dichter des 19. Jahrhunderts, Charles Baudelaire, in seinen Causeries du lundi jedoch gar nicht behandelt und der z. B. von Stendhal kurz und bündig sagt: „les romans de Stendhal [...] sont franchement détestables.“ (S. 138) Literarische Werke sind für Proust Ergebnisse der Beschäftigung eines Autors mit sich selbst; sie sind Produkte der Stille: „Les livres sont l'œuvre de la solitude et les enfants du silence.“ (S. 309) Der Stoff und die Sujets einer wertigen Literatur dürfen nicht einfach die Wirklichkeit „telle quelle“ widerspiegeln; sie sind - hier spielt Proust auf die in der Recherche hervortretenden ,mémoires involontaires' an - auf eine epiphanische, gleichsam ,immaterielle“ Weise entstehende Einblicke in die Substanz und Tiefe des Ichs.

La matière de nos livres, la substance de nos phrases doit être immatérielle, non pas prise telle quelle dans la réalité, mais nos phrases elles-mêmes, et les épisodes aussi doivent être faits de la substance transparente de nos minutes les meilleures, où nous sommes hors de la réalité et du présent. C'est de ces gouttes de lumière cimentées que sont faits le style et la fable d'un livre. (S. 309)

Literatur mit einer derartigen Tiefendimension will sich von den sozialen Gepflogenheiten des Lebens befreien. Sie lässt die aus der alltäglichen Konversation bekannten vorgefertigten Ausdrucksweisen (,expressions toutes faites“) und die Masken, die der Mensch des sozialen Alltagslebens trägt, hinter sich:

[...] d'abord qu'il y ait profondeur, qu'on ait atteint les régions de la vie spirituelle où l'œuvre d'art peut se créer. Or, quand nous verrons un écrivain à chaque page, à chaque situation où se trouve son personnage, ne jamais l'approfondir, ne pas le repenser sur lui-même, mais se servir des expressions toutes faites, que ce qui en nous vient des autres - et des plus mauvais autres - nous suggère quand nous voulons parler d'une chose, si nous ne descendons pas dans ce calme profond où la pensée choisit les mots où elle se reflétera tout entière; un écrivain qui ne voit pas sa propre pensée, alors invisible à lui, mais se contente de la grossière apparence qui la masque à chacun de nous à tout moment de notre vie, dont le vulgaire se contente dans une perpétuelle ignorance, et que l'écrivain écarte, cherchant à voir ce qu'il y a au fond [...] (S. 306f.)

Wahre Literatur hat für Proust nichts mit der Konversation zu tun, in der eine Person durchaus intime Dinge einer bestimmten Zahl von Adressaten vermittelt, die aber zur äußerlichen Rolle der gesellschaftlichen Existenz gehören und nicht 
zum ,moi profond' des Schriftstellers, welcher von der sozialen Rolle abstrahieren muss:

\begin{abstract}
En réalité, ce qu'on donne au public, c'est ce qu'on a écrit seul, pour soi-même, c'est bien l'œuvre de soi. Ce qu'on donne à l'intimité, c'est-à-dire à la conversation [...] et ces productions destinées à l'intimité, c'est-à-dire rapetissées au goût de quelques personnes et qui ne sont guère que de la conversation écrite, c'est l'œuvre d'un soi bien plus extérieur, non pas du moi profond qu'on ne retrouve qu'en faisant abstraction des autres et du moi qui connaît les autres, le moi qui a attendu pendant qu'on était avec les autres, qu'on sent bien le seul réel, et pour lequel seuls les artistes finissent par vivre, comme un dieu [...] à qui ils ont sacrifié une vie qui ne sert qu’à l'honorer. (S. 141)
\end{abstract}

Es ist die Unbedingtheit dieser poetologischen Position, die den Leser der späteren Recherche angesichts der so zahlreichen Abbildungen der Konversationen irritiert. Ulrich Schulz-Buschhaus hat gezeigt, dass Proust anders als sein Vorbild Flaubert die abgebildeten Konversationen mit ihren vorgestanzten Formeln und ihren ,idées reçues“ nie durch eine „distanzierende Gesprächsregie“ erzählpragmatisch reduziert. Er verzichtet auf diese Weise darauf, ihnen eine Funktion in der Syntagmatik des Erzählten zuzuweisen. Schulz-Buschhaus wertet dies als Zeichen dafür, wie weit der konversationskritische Proust von den Gesprächen der Salons abhängig war.

\title{
14.3 Prousts Sezierung der Kunst des Impliziten in der Konversation
}

Die Romane von Prousts $A$ la Recherche du temps perdu sind in erster Linie minutiöse Aufzeichnungen von Begegnungen des Erzählers Marcel mit Figuren der Aristokratie und des Bürgertums in diversen Salons seiner Zeit, die Marcel dann ebenso minutiös analysiert. So sehr sich dieser Erzähler innerlich gegen die sozialen Räume der gesellschaftlichen Konversation auflehnt, da er sich als Schriftsteller das Ziel der Erforschung der Tiefe des eigenen Ichs gesetzt hat, so sehr ist er auf die Begegnungen in diesen sozialen Räumen angewiesen, allein, weil er dort seinen Stoff findet und seine Analysekriterien in der Kritik der von ihm zurückgewiesenen Konversation schärfen kann. Im zweiten Roman der Recherche, A l'ombre des jeunes filles en fleurs (1918), beschreibt Marcel wie er vergeblich versucht, dem Sog der gesellschaftlichen Salonkonversation zu entrinnen, und wie er immer wieder von den dortigen Begegnungen angezogen wird. Anstatt sich der Erforschung des Ichs in der Einsamkeit und Stille seines heimischen Schreibtischs zu widmen, führt Marcel imaginäre Gespräche mit den Teilnehmern der von ihm frequentierten Salons, um Pointen vorzubereiten, die seinem Auftreten in der Ge- 
sellschaft ,esprit‘ verleihen. Statt das Ich zu ergründen, entpuppt sich sein Inneres als ,salon mental':

[...] quand leur demeure [= des Swanns] me fut ouverte, à peine je m'étais assis à mon bureau de travail que je me levais et courais chez eux. Et une fois que je les avais quittés et que j'étais rentré à la maison, mon isolement n'était qu'apparent, ma pensée ne pouvait plus remonter le courant du flux de paroles par lequel je m'étais laissé machinalement entraîner pendant des heures. Seul je continuais à fabriquer les propos qui eussent été capables de plaire aux Swann et pour donner plus d'intérêt au jeu, je tenais la place de ces partenaires absents, je me posais à moi-même des questions fictives choisies de telle façon que mes traits brillants ne leur servissent que d'heureuse repartie. Silencieux, cet exercice était pourtant une conversation et non une méditation, ma solitude une vie de salon mentale où c'était non ma propre personne mais des interlocuteurs imaginaires qui gouvernaient mes paroles et où j'éprouvais à former, au lieu des pensées que je croyais vraies celles qui me venaient sans peine, sans régression du dehors vers le dedans, ce genre de plaisir tout passif que trouve à rester tranquille quelqu'un qui est alourdi par une mauvaise digestion..$^{29}$

Kreative Schreibprozesse entstehen für Proust aus dem Gefühl und nicht aus dem Verstand heraus. Solche - wie es heißt - „wahren Gedanken“, die „spontan aus dem Innern emporkommen“ („qui me venaient sans peine“), sind ihm im inneren Gespräch mit den imaginären Konversationspartnern verwehrt, da die Gespräche seine eigenen Worte dominieren. Der Vergleich dieser „vie de salon mentale“ mit dem zweifelhaften Vergnügen einer schlechten Verdauung zeigt, was Marcel von der sozialen Konversation hält. Die Salonkonversation zwingt dem Einzelnen Rollen auf. Sie verformt seine Sprache („des interlocuteurs imaginaires qui gouvernaient mes paroles“). Allein dadurch gewinnt sie Dimensionen einer indirekten Kommunikation: Die Konversation ist für Proust nur Täuschung und Selbsttäuschung ${ }^{30}$.

Die Romane Prousts konzentrieren sich wesentlich auf die Analyse solcher Verformungen, die die soziale Sphäre letztlich als Lügengebäude erweist. Ihm geht es um die Beschreibung der gesprochenen Sprache und ihrer situtationsgebundenen Deformation. Der Erzähler erfasst genauestens die Sprachtstile der Figuren und den ihnen zugrunde liegenden jeweiligen Habitus ${ }^{31}$. Für Marcels Sezierung der Konversationspraxis liefert ein Beispiel besonders prägnante Einsichten: der Blick auf den Geist der aristokratischen Familie der Guermantes („l'esprit des

29 Marcel Proust, À l'ombre des jeunes filles en fleurs I, in: M. P., À la recherche du temps perdu, 4 Bde., hrsg. von Jean-Yves Tadié (Bibliothèque de la Pléiade. 100. 101. 102. 356) Paris, Gallimard, 1987-1989, Bd. 1, S. 421-630, hier: S. 569.

30 Vgl. dazu Serge Gaubert, „La Conversation et l'écriture“, Europe Bd. 496/1970, S. 171-192, bes. S. 174.

31 Vgl. Gaubert, „La Conversation“, S. 171 und S. 176 f. 
Guermantes"), und ganz besonders auf die Duchesse Oriane de Guermantes, die den Stil der französischen Konversation auf eine für Proust typische Weise verkörpert.

Der ,esprit des Guermantes' ist insbesondere Thema des dritten Romans der Recherche, Le Côté de Guermantes (1920/1921). Der Roman spielt im ,Quartier Saint-Germain' in Paris, wo Marcel in der Nähe der Familie der Guermantes wohnt. Seit seiner Kindheit ist der Name dieser Adelsfamilie für ihn geradezu mythisch besetzt. Nach einem Opernbesuch verliebt er sich in die Herzogin, der er daraufhin bei seinen morgendlichen Spaziergängen immer wieder zu begegnen sucht. Bei einer Einladung in den Salon der Madame de Villeparisis, der Tante der Duchesse de Guermantes, trifft er zum ersten Mal direkt auf seine Liebe, wird aber im Laufe des Abends durch die Art und Weise ihrer Konversation von ihr maßlos enttäuscht. Als er sich längst einer anderen Liebe zugewandt hat, lädt ihn die Duchesse zu seiner Überraschung zu einem Empfang in ihr Haus in Saint-Germain ein. Diesen Höhepunkt des Romans nutzt der Autor, um die zeitgenössische Aristokratie zu groteskisieren: Der Erzähler Marcel seziert die Gesellschaft mit messerscharfem Blick und entlarvt insbesondere das Verhalten der Mme de Guermantes als rollenhafte Verstellung.

Gleich zu Beginn des Romans wird die Absicht Marcels erkennbar, den Mythos um die Protagonistin, zugleich Mittelpunkt der Familie derer von Guermantes, zu ergründen. Nach dem mittelalterlichen realistischen Prinzip ,nomina sunt consequentia rerum ' sucht Marcel anfänglich in ihrem klangvollen Namen die tiefere Bedeutung der Familie, die der französischen Hocharistokratie entstammt. Es wird aber auch bereits deutlich, dass der Protagonist dabei nur desillusioniert werden kann

\footnotetext{
Parfois, cachée au fond de son nom, la fée se transforme au gré de la vie de notre imagination qui la nourrit; c'est ainsi que l'atmosphère où Mme de Guermantes existait en moi, après n'avoir été pendant des années que le reflet d'un verre de lanterne magique [...]

Cependant, la fée dépérit si nous nous approchons de la personne réelle à laquelle correspond son nom, car, cette personne, le nom alors commence à la refléter et elle ne contient rien de la fée $[\ldots]^{32}$ (S. 6 f.)
}

Der bis dahin nur mit Büchern vertraute Marcel muss die in der Recherche immer wieder angesprochene Erfahrung machen, dass die Aussagen seiner Gesprächspartner in den Salonkonversationen nicht auf direktem Wege erfolgen. In Verbindung mit der weitgehend unkontrolliert und willkürlich erfolgenden Körperspra-

32 Le Côté de Guermantes, in: M. P., À la recherche du temps perdu, Bd. 2, 307-884, hier: S. 311. Die Stellenangaben im laufenden Text beziehen sich auf diese Ausgabe. 
che des Gegenübers lässt sich der Sinn der Äußerungen hinter der Verstellung interpretieren:

[...] moi qui pendant tant d'années, n’avais cherché la vie et la pensée réelles des gens que dans l'énoncé direct qu'ils m'en fournissaient volontairement, par leur faute j'en étais arrivé à ne plus attacher, au contraire, d'importance qu'aux temoignages qui ne sont pas une expression rationnelle et analytique de la vérité; les paroles elles-mêmes ne me renseignaient qu'à la condition d'être interpretées à la façon d'un afflux de sang à la figure d'une personne qui se trouble, à la façon encore d'un silence subit. ${ }^{33}$

Mme de Guermantes wird nun mit allen Mitteln der sprachlichen und körpersprachlichen Analyse entschlüsselt. Sie erscheint Marcel als Inkaration der alten Aristokratie, die unter Ludwig XIV. ihren kulturellen Höhepunkt hatte. Durch die zivilisatorische äußere Hülle ihrer Liebenswürdigkeit und ihrer spirituellen Werte hindurch vermag der Erzähler in ihrer stets angriffslustigen Ansprache (,verve frondeuse“, S. 735) das Verhalten der alten Landaristokratie zu erkennen:

Mme de Guermantes m'offrait, domestiquée et soumise par l'amabilité, par le respect envers les valeurs spirituelles, l'énergie et le charme d'une cruelle petite fille de l'aristocratie des environs de Combray, qui, dès son enfance, montait à cheval, cassait les reins aux chats, arrachait l'œil aux lapins [...] (S. 793)

Die an den Tag gelegte Höflichkeit der Guermantes sowie ihr Benehmen faszinieren den Protagonisten, der darin die Sitten und Gebräuche des 17. Jahrhundert wiedererkennt („d'habitudes en particulier du XVII ${ }^{\mathrm{e}}$ siècle“, S. 710). Marcel geht diesen Verhaltensweisen, die ihn insbesondere bei dem oftmals so merkwürdig vulgär auftretenden M. de Guermantes auffallen und beeindrucken (,cette politesse de M. de Guermantes [...] me charma comme un reste d'habitudes [...] séculaires“, ebd.) auf den Grund. Er erkennt, dass es sich bei diesen „marques de courtoisie“ um verinnerlichte Einstellungen handelt, die die Personen beherrschen und sie dazu bringen, stets grundsätzlich Höflichkeit und Liebenswürdigkeit vorzutäuschen:

[...] chez tels personnages de la cour de Louis XIV, quand nous trouvons des marques de courtoisie dans des lettres écrites par eux à quelque homme de rang inférieur et qui ne peut leur être utile à rien, elles nous laissent surpris parce qu'elles nous révèlent tout à coup chez ces grands seigneurs tout un monde de croyances qu'ils n'expriment jamais directement mais qui les gouvernent, et en particulier la croyance qu'il faut par politesse feindre certains sentiments et exercer avec le plus grand scrupule certaines fonctions d'amabilité. (Ebd.)

33 La Prisonnière, in: M. P., À la recherche du temps perdu, Bd. 3, S. 517-915, hier: S. 596. 
Insbesondere geht Marcel dem Phänomen der Habitualisierung solcher Verhaltensweisen nach und erkennt ganz im Sinn der sich wenig später entwickelnden Mentalitätsgeschichte, wie die entsprechenden Einstellungen entstehen, sich verfestigen und über lange Zeiträume (,longue durée') fortbestehen:

[...] le savant qui étudie dans une région lointaine la toponymie, les coutumes des habitants, pourra saisir encore en elles telle légende bien antérieure au christianisme, déjà incomprise, sinon même oubliée, au temps d'Hérodote et qui, dans l'appellation donnée à une roche, dans un rite religieux, demeuré au milieu du présent comme une émanation plus dense, immémoriale et stable. Il y en avait une aussi, bien moins antique, émanation de la vie de cour, sinon dans les manières souvent vulgaires de M. de Guermantes, du moins dans l'esprit qui les dirigeait. Je devais la goûter encore, comme une odeur ancienne, quand je le retrouvai un peu plus tard au salon. (S. 711)

Im Fall der Duchesse de Guermantes führen die Beobachtungen Marcels zu einer großen Enttäuschung, insbesondere als er der Frage nachgeht, ob die Duchesse, wie gemeinhin angenommen wird, besonders intelligent ist und daher eine ,profondeur' unter Beweis stellt:

[...] j'avais peine à retrouver dans le beau visage, trop humain, de Mme de Guermantes, l'inconnu de son nom, je pensais du moins que, quand elle parlerait, sa causerie, profonde, mystérieuse, aurait une étrangeté de tapisserie médiévale, de vitrail gothique. (S. 506)

Letztlich kommt der Erzähler zu dem Ergebnis, dass es sich nicht um die Intelligenz großer Geister handelt: „ce n'est nullement l'intelligence telle que je la connaissais [...] fût-ce celle des plus grands esprits“ (S. 507). Die Intelligenz der Duchesse de Guermantes richtet sich auf die „conversation insignifiante“ (ebd.), auf die Banalitäten des Alltags sowie die ,maldisances‘ über allen Gesprächsteilnehmern bekannte, zumeist nicht anwesende Personen. Eine - ausnahmsweise kurze Konversationsszene zwischen Oriane und ihrer Tante, Mme de Villeparisis, vermag diesen ,esprit‘ der Mme de Guermantes zu verdeutlichen:

„Je croyais trouver Basin [= M. de Guermantes] ici, il comptait venir vous voir, dit Mme de Guermantes à sa tante.

- Je ne l'ai pas vu, ton mari, depuis plusieurs jours, répondit d'un ton susceptible et fâché Mme de Villeparisis. Je ne l'ai pas vu, ou enfin peut-être une fois, depuis cette charmante plaisanterie de se faire annoncer comme la reine de Suède.“

Pour sourire Mme de Guermantes pinça le coin de ses lèvres comme si elle avait mordu sa voilette.

„Nous avons dîné avec elle hier chez Blanche Leroi, vous ne la reconnaîtriez pas, elle est devenue énorme, je suis sûre qu'elle est malade.

- Je disais justement à ces messieurs que tu lui trouvais l'air d'une grenouille.“

Mme de Guermantes fit entendre une espèce de bruit rauque qui signifiait qu'elle ricanait par acquit de conscience. 
„Je ne savais pas que j'avais fait cette jolie comparaison, mais, dans ce cas, maintenant c'est la grenouille qui a réussi à devenir aussi grosse que le bœuf. Ou plutôt ce n'est pas tout à fait cela, parce que toute sa grosseur s'est amoncelée sur le ventre, c'est plutôt une grenouille dans une position intéressante.

- Ah! je trouve ton image drôle“, dit Mme de Villeparisis qui était au fond assez fière pour ses visiteurs de l'esprit de sa nièce. (S. 507 f.; Hervorhebungen von Vf.)

Die kurze Konversation ist durch und durch der Kunst des Impliziten verpflichtet und zugleich von einer unfreiwilligen Komik: Die Bemerkung der Duchesse de Guermantes, Blanche Leroi sehe aufgrund ihres Leibesumfangs mittlerweile aus wie der Frosch, der sich zum Ochsen aufbläht, wird von Ihrer Tante als Beweis des ,esprit' der Nichte angesehen, den sie ihren Salongästen nur allzu gerne vorführt. Der Vergleich stellt schließlich eine literarische Bildung unter Beweis, erinnert das Bild vom sich aufblasenden Frosch doch an La Fontaines Fabel La Grenouille qui se veut faire aussi grosse que le bœuf. Wenn Mme de Guermantes jedoch en passant sagt, sie sei beim Empfang der Blanche Leroi auf die Königin von Schweden getroffen, stiehlt sie ihrer Tante die Schau, wollte diese doch wie der Frosch in der Fabel per ,name-dropping i ihren Salon aufwerten ${ }^{34}$, der ab und zu von der Königin von Schweden frequentiert wird. Nun ist der Forsch, der sich aufbläst, vor allem die Duchesse selbst, dient ihr die ,maldisance' gegen Mme Leroi doch nur dazu, ihre Bekanntschaft mit der Königin von Schweden herauszustellen. Ihre körpersprachliche Äußerung - „une espèce de bruit rauque“ - lässt sie hörbar zum ,aufgeblasenen Frosch` werden.

Die Illusion des Namens der Guermantes ist für den Erzähler schon lange wie der Frosch der Fabel geplatzt. Die Duchesse kommt über das Froschquaken nicht hinaus. Ihre vermeintliche Intelligenz ist in Wirklichkeit eine antrainierte Attitude, mit der sie in der Öffentlichkeit ihren ,esprit' unter Beweis zu stellen sucht. Mme de Guermantes produziert am laufenden Band Paradoxien bzw. überraschende Äußerungen, die sich dem gewöhnlichen Teilnehmer einer Konversation nicht erschließen, jedoch Intelligenz und Schlagfertigkeit simulieren. Besonders gilt dies im Fall der Diskussion über Literatur, beansprucht Oriane de Guermantes doch eine gewisse literarische Bildung für sich. Ihre entsprechenden Äußerungen werden mit den Kriterien Sainte-Beuves in Verbindung gebracht, hatte dieser ja für Proust ebenfalls stets - konversationstypisch - auf persönliche Dinge gesetzt und die Charakterisierung eines Autors auf höchst subjektive Eingebungen gestützt. In einer Diskussion mit der Princesse de Parme lassen sich diese Kriterien erkennen:

34 Basin hatte seinerzeit den Spaß gemacht, sich bei Mme de Villeparisis als Königin von Schweden anzukündigen. 
Quant aux actions mondaines, c'était encore un autre plaisir arbitrairement théâtral que Mme de Guermantes éprouvait à émettre sur elles de ces jugements imprévus qui fouettaient de surprises incessantes et délicieuses la princesse de Parme. (S. 763 f.)

Wie ein alter ego von Sainte-Beuve überrascht die Duchesse die verdutzte Princesse de Parme mit ihrer Einschätzung, Émile Zola sei keineswegs ein realistischer Erzähler sondern ein Dichter, der alles was er anfasse, groß mache wie Homer. Er sei - so ihre grotesken Kriterien - der „Homer der Fäkalien“ und besitze „epischen Dünger" :

„[...] Zola n’est pas un réaliste, Madame! c'est un poète!“ dit Mme de Guermantes, s’inspirant des études critiques qu'elle avait lues dans ces dernières années et les adaptant à son génie personnel. Agréablement bousculée jusqu'ici, au cours du bain d'esprit, un bain agité pour elle, qu'elle prenait ce soir, et qu'elle jugeait devoir lui être particulièrement salutaire, se laissant porter par les paradoxes qui déferlaient l'un après l'autre, devant celui-ci, plus énorme que les autres, la princesse de Parme sauta par peur d'être renversée. Et ce fut d'une voix entrecoupée, comme si elle perdait sa respiration, qu'elle dit:

„Zola, un poète!

- Mais oui“, répondit en riant la duchesse, ravie par cet effet de suffocation. „Que Votre Altesse remarque comme il grandit tout ce qu'il touche. Vous me direz qu'il ne touche justement qu'à ce qui... porte bonheur! Mais il en fait quelque chose d'immense; il a le fumier épique! C’est l’Homère de la vidange! [...]““ (S. 789)

Die Konversation im Salon ist für Mme de Guermantes ein Spiel, mit dem sie Wertvorstellungen ihrer Gesprächspartner umstürzen will (,Mme de Guermantes renversait sans cesse l'ordre des valeurs chez les personnes de son milieu [...]“, S. 764). Auf diese Weise sucht sie sich an den emotionalen Turbulenzen der Abendgesellschaft zu ergötzen (,à goûter ces émotions artificielles [...] qui stimulent la sensibilité des assemblées“, ebd.). Dies zeigt für Proust, wie gehaltlos die Konversation in Frankreich um die Wende zum 20. Jahrhundert geworden ist. Aus der vormals geistvollen Unterhaltung zum Zweck der Geselligkeit gebildeter Gesprächspartner ist ein rituelles Spiel aus willkürlich gesetzten Pointen und ,traits d'esprit' geworden, deren ,esprit' sich in Boshaftigkeiten und nur pseudointelligenten Wendungen ergeht. Für Proust, der auf der Suche nach den Tiefen des Ichs ist, gilt dies für jede Form der Salonkonversation, ob im 17., 18. oder 19. Jahrhundert, die Sainte-Beuve zum Vorbild seiner literarischen Kritik gemacht hatte und die der Autor der Recherche für nichtig hält:

[...] lorsqu'un peintre véridique comme Sainte-Beuve veut marquer successivement les nuances qu'il y eut entre le salon de Mme Geoffrin, de Mme Récamier et de Mme de Boigne, ils apparaissent tous si semblables que la principale vérité qui, à l'insu de l'auteur, ressort de ses études, c'est le néant de la vie de salon [...] (S. 709) 
Bei aller Widerständigkeit gegen die Konversation und die ,dissimulatio“ durch eine indirekte Sprache und Körpersprache, ist die Inszenierung des ,moi profond" bei Proust schon in der Schrift gegen Sainte-Beuve jedoch gleichwohl an ein Gespräch gebunden. Die entsprechenden Ausführungen zur schriftstellerischen Endeckung des ,moi profond' und seiner Fixierung der Entdeckungen in Contre Sainte-Beuve entwickelt Marcel im Gespräch mit seiner Mutter. Sie sind damit ein Stück inszenierter Mündlichkeit. Die konstruierte Opposition von ,moi profond' und ,moi social' wird durch diese inszenierte Mündlichkeit unterlaufen, was sich für die Recherche an zahlreichen Stellen zeigen lässt. Dies zeigt, dass sich Proust der so heftig kritisierten Konversation und ihrer Kunst des Impliziten nicht entziehen kann:

Die Teilnahme an der Konversation ist nicht bloßes Hindernis auf dem Weg zum wahren Kunstwerk, durch die Verwicklung sowohl des erlebenden als auch des erzählenden Ich in das snobistische Spiel mit der Sprache werden beide vielmehr so weit zu deren Objekt, daß der erzählerische Diskurs selbst davon affiziert wird. ${ }^{35}$

35 Sprenger, Stimme und Schrift, S. 78. Vgl. auch S. 5 und S. 12. 